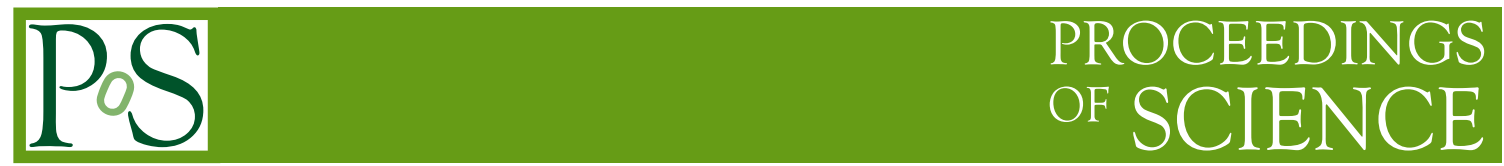

\title{
Measurement of the CP-violating phase $\phi_{s}$ in the $B_{s}^{0} \rightarrow J / \psi \phi$ channel at $\sqrt{s}=13 \mathrm{TeV}$ by CMS
}

\section{Alberto Bragagnolo,* for the CMS collaboration}

University of Padova \& INFN Sezione di Padova,

Via Francesco Marzolo 8, Padova, Italy

E-mail: alberto.bragagnolo@pd.infn.it

These proceedings present the measurement of the weak CP-violating phase $\phi_{s}$ in the $B_{s}^{0} \rightarrow$ $J / \psi \phi(1020)$ decay channel at $\sqrt{s}=13 \mathrm{TeV}$ with the CMS experiment. The measurement is based on a data sample corresponding to an integrated luminosity of $96.4 \mathrm{fb}^{-1}$, collected in 2017-2018. This analysis employs a dedicated tagging trigger and a novel opposite-side muon flavor tagger based on deep learning techniques. The decay width (mass) difference $\Delta \Gamma_{s}\left(\Delta m_{s}\right)$ between the light and heavy $B_{s}^{0}$ mass eigenstates, the $B_{s}^{0}$ average decay width $\Gamma_{s}$ and the CP violation observable $|\lambda|$ are also measured. The combination with the results obtained by CMS at $\sqrt{s}=8 \mathrm{TeV}$ is also presented.

The Eighth Annual Conference on Large Hadron Collider Physics-LHCP2020

25-30 May, 2020

online

${ }^{*}$ Speaker 


\section{Introduction}

The CP-violating (CPV) phase $\phi_{s}$ arises from the interference between $B_{s}^{0}$ decays proceedings directly and through $B_{s}^{0}-\bar{B}_{s}^{0}$ mixing to a CP eigenstate. In the Standard Model (SM), $\phi_{s}$ is related to the elements of the CKM matrix via $\phi_{s} \simeq-2 \beta_{s}=-2 \arg \left(-V_{\mathrm{ts}} V_{\mathrm{tb}}^{*} / V_{\mathrm{cs}} V_{\mathrm{cb}}^{*}\right)$, where $\beta_{s}$ is one of the angles of the unitary triangles. Assuming no physics beyond the SM (BSM) in the $B_{s}^{0}$ mixing and decays, a $-2 \beta_{s}$ value of $-36.96_{-0.84}^{+0.72} \mathrm{mrad}$ is determined [1]. New Physics (NP) can change the value of $\phi_{s}$ up to $\approx 10 \%$ via new particles contributing to the $B_{s}^{0}-\bar{B}_{s}^{0}$ mixing [2]. $B_{s}^{0} \rightarrow J / \psi \phi$ is the golden channel to measure $\phi_{s}$, since it presents no direct predicted CPV and only one CPV phase. This channel also allows measurements of several other interesting observables, such as the decay width (mass) difference $\Delta \Gamma_{s}\left(\Delta m_{s}\right)$ between the light and heavy $B_{s}^{0}$ mass eigenstates, the $B_{s}^{0}$ average decay width $\Gamma_{s}$ and the direct $\mathrm{CP}$ violation observable $|\lambda|$. These proceedings presents the latest measurement of $\phi_{s}$ by CMS, performed in proton-proton (pp) collisions at $\sqrt{s}=13 \mathrm{TeV}$ [3]. The measurement is performed with a time-dependent and flavor-tagged angular analysis of the $\mu^{+} \mu^{-} K^{+} K^{-}$final state. Since the final state is mixture of CP eigenstates an angular analysis is used to separate the CP-odd and CP-even components. This measurement uses the transversity basis defined by the three decay angles $\theta_{T}, \psi_{T}$, and $\phi_{T}$ [4].

\section{Candidate selection}

The analysis is performed using data collected in pp collisions at $\sqrt{s}=13 \mathrm{TeV}$ during 20172018, corresponding to an integrated luminosity of $96.4 \mathrm{fb}^{-1}$. For this measurement a trigger that requires a $J / \psi \rightarrow \mu^{+} \mu^{-}$candidate plus an additional (third) muon is used. The additional muon can be used to infer the flavor of the $B_{s}^{0}$, via $b \rightarrow \mu^{-} X$ decays of the other b hadron in the event. The requirement for a third muon, however, lowers the rate of selected events. This trigger strategy improves the fraction of tagged events (tagging efficiency) at the cost of a reduced number of signal events. As a result, this measurement, while based on a similar number of $B_{s}^{0}$ candidates as the previous CMS measurement [5], doubles the precision in the determination of $\phi_{s}$. At the same time, the precision on parameters that do not benefit from the tagging information, such as $\Delta \Gamma_{s}$, is comparable to that in the previous measurement. Candidates are further selected with several quality cuts optimized to maximize the signal purity $N_{\text {sig }} /\left(N_{\text {sig }}+N_{\text {bkg }}\right)$. A total of 65500 $B_{s}^{0} \rightarrow J / \psi \phi$ candidates are selected.

\section{Flavor tagging}

To infer the $B_{s}^{0}$ flavor at production time, an opposite side (OS) muon tagging algorithm has been developed in $B_{s}^{0} \rightarrow J / \psi \phi$ simulated events and calibrated in data using $B^{ \pm} \rightarrow J / \psi K^{ \pm}$selftagging decays. The tagging muon candidate is selected with loose kinematic requirements, after excluding the b meson candidate tracks. To reduce the background from light hadrons (mainly $K^{ \pm}$ and $\pi^{ \pm}$) an MVA discriminator has been trained with muon candidates from simulated samples. The choice of requiring the muon already at trigger level, combined with the loose selection, leads to a very high tagging efficiency of about $\epsilon_{\text {tag }}=N_{\text {tagged }} / N_{\text {tot }} \approx 50 \%$. The mistag probability $\omega_{\text {tag }}=N_{\text {mistagged }} / N_{\text {tagged }}$ is evaluated on per-event basis with a dedicated fully connected Deep 
Neural Network, constructed in such a way that the output score $f_{\mathrm{dnn}}$ is equal to the probability of tagging the event correctly. The mistag probability is then easily computed as $\omega_{\text {tag }}=1-f_{\text {dnn }}$ and it is calibrated in data with self-tagging $B^{ \pm} \rightarrow J / \psi K^{ \pm}$decays. A tagging efficiency of $\approx 50 \%$ and a tagging power of $\approx 10 \%$ are achieved in both the 2017 and 2018 data samples.

\section{Maximum likelihood fit}

The differential decay rate is described by time-dependent and angular functions [6]. Possible contributions of $B_{s}^{0} \rightarrow J / \psi f_{0}(980)$ and non resonant $B_{s}^{0} \rightarrow J / \psi K^{+} K^{-}$decays are taken into account by including a term for an additional $S$-wave amplitude in the decay model. The fit model also includes the background parametrization, which contains two terms to model both the combinatorial background and the peaking background, dominated by $B^{0} \rightarrow J / \psi K^{* 0} \rightarrow$ $\mu^{+} \mu^{-} K^{+} \pi^{-}$. Efficiency functions model the dependence of the signal reconstruction efficiency on the proper decay length and the three angles of the transversity basis. An unbinned multidimensional extended maximum-likelihood fit is performed on the combined data samples using 8 observables as input: the $B_{s}^{0}$ candidate invariant mass, the three decay angles of the reconstructed $B_{s}^{0} \rightarrow J / \psi \phi$ candidate, the flavor tag decision, the mistag fraction, the proper decay length of the $B_{s}^{0}$ candidate, and its uncertainty. The distributions of the input observables and the corresponding fit projections are shown in Fig. 1. The measured number of $B_{s}^{0} \rightarrow J / \psi \phi$ signal events from the fit is $48500 \pm 250$.

\section{Results}

The CPV phase is measured to be $\phi_{s}=-11 \pm 50$ (stat) \pm 10 (syst) mrad, while the decay width difference is measured $\Delta \Gamma_{s}=0.114 \pm 0.014$ (stat) \pm 0.007 (syst) $\mathrm{ps}^{-1}$. The $|\lambda|$ parameter is measured to be $|\lambda|=0.972 \pm 0.026$ (stat) \pm 0.008 (syst), consistent with no direct CP violation $(|\lambda|=1)$. The average of the heavy and light $B_{s}^{0}$ mass eigenstate decay widths is determined to be $\Gamma_{s}=0.6531 \pm 0.0042$ (stat) \pm 0.0024 (syst) $\mathrm{ps}^{-1}$, consistent with the world-average value [7]. The mass difference between the heavy and light $B_{s}^{0}$ meson mass eigenstates is measured to be $\Delta m_{s}=17.51_{-0.09}^{+0.10}$ (stat) \pm 0.02 (syst) $\hbar \mathrm{ps}^{-1}$, consistent with the theoretical prediction [8], and in slight tension with the world-average value [7]. The uncertainties in all these measured parameters are dominated by the statistical component. This analysis represents the first measurement by CMS of the mass difference $\Delta m_{s}$ and of the direct $\mathrm{CP}$ observable $|\lambda|$. The results are in agreement with the earlier CMS result at a center-of-mass energy of $8 \mathrm{TeV}$ [5] and therefore combined. The two sets of results are combined using their respective correlation matrices, with their respective systematic uncertainties treated as uncorrelated. The combined results for the CP-violating phase and lifetime difference between the two mass eigenstates are:

$$
\begin{gathered}
\phi_{s}=-21 \pm 45 \mathrm{mrad}, \\
\Delta \Gamma_{s}=0.1073 \pm 0.0097 \mathrm{ps}^{-1} .
\end{gathered}
$$

The two-dimensional $\phi_{s}$ vs. $\Delta \Gamma_{s}$ likelihood contours at $68 \%$ confidence level (CL) for the individual and combined results, as well as the SM prediction, are shown in Fig. 2. The results are in agreement with each other and with the SM predictions. 

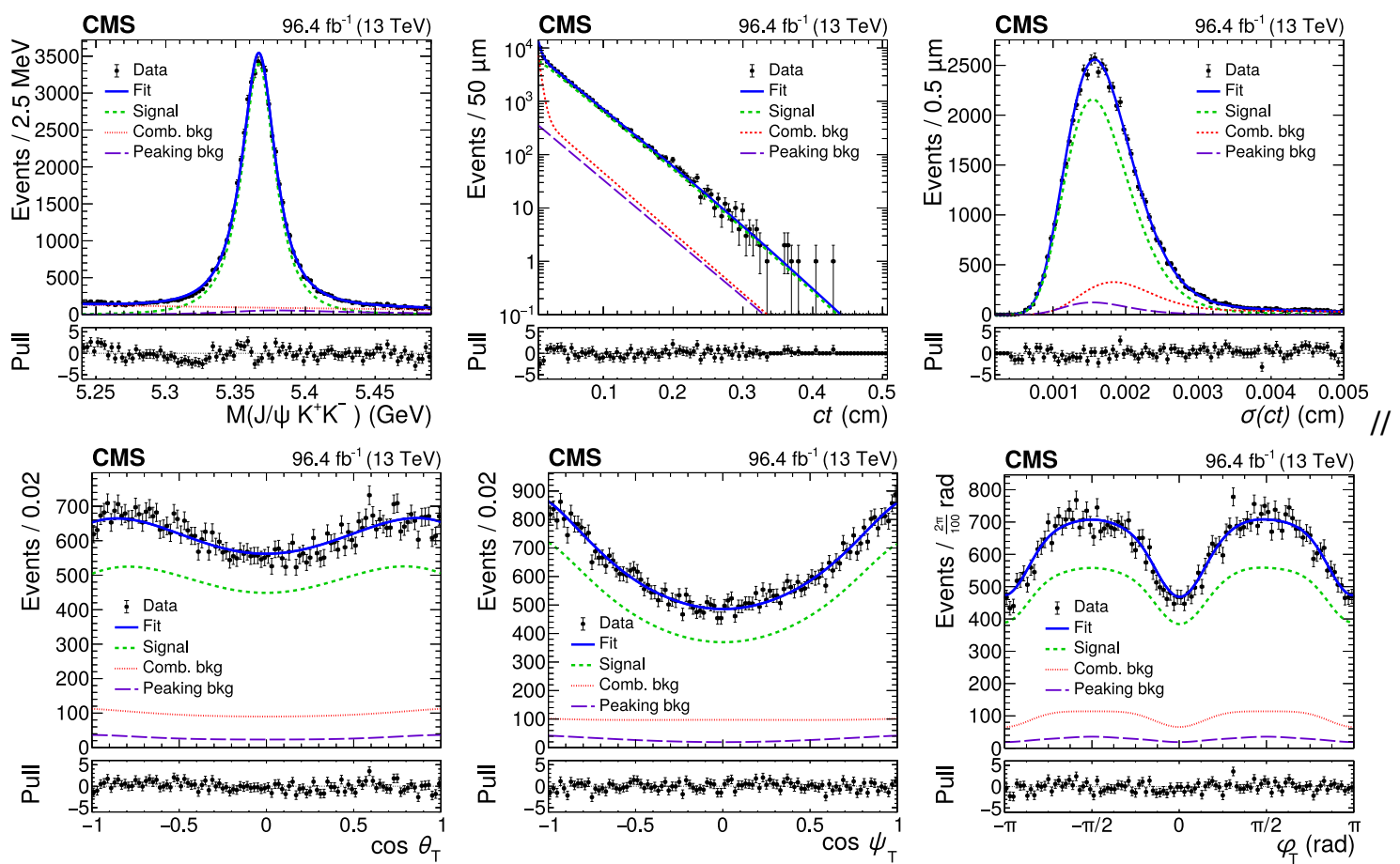

Figure 1: The invariant mass distribution (top left), $c t$ distribution (top middle), its uncertainty (top right), and the angular distributions $\cos \theta_{T}$ (bottom left), $\cos \psi_{T}$ (bottom middle) and $\phi_{T}$ (bottom right) for the $B_{s}^{0} \rightarrow J / \psi \phi \rightarrow \mu^{+} \mu^{-} K^{+} K^{-}$candidates in data. The blue line represents the fit to data projection. Figure from [3].

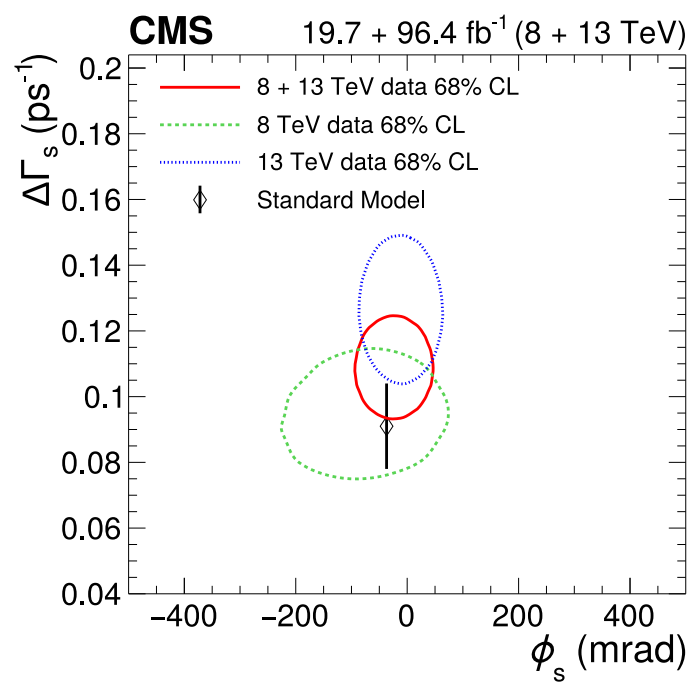

Figure 2: The 2D likelihood contours at $68 \% \mathrm{CL}$ for the $\mathrm{CMS} 8 \mathrm{TeV}$ (dashed line), $13 \mathrm{TeV}$ (dotted line), and combined (solid line) results. The SM prediction is shown with the diamond marker [1, 8]. Figure from [3]. 


\section{References}

[1] The CKM fitter Group Collaboration, Predictions of selected flavour observables within the Standard Model, Phys. Rev. D 84 (2011) 033005.

[2] C. W. Chiang et al., New physics in $B_{s}^{0} \rightarrow J / \psi \phi:$ a general analysis, JHEP 04 (2010) 031 [hep-ph/0910.2929].

[3] CMS Collaboration, Measurement of the CP violating phase $\phi_{s}$ in the $B_{s}^{0} \rightarrow J / \psi \phi \rightarrow$ $\mu^{+} \mu^{-} K^{+} K^{-}$channel in proton-proton collisions at $\sqrt{s}=13 \mathrm{TeV}$, CMS-BPH-20-001, [hep-ex/2007.02434].

[4] A. S. Dighe, I. Dunietz, and R. Fleischer, Extracting CKM phases and $B_{s}^{0}-\bar{B}_{s}^{0}$ mixing parameters from angular distributions of non-leptonic B decays, Eur. Phys. J. C 6 (1999) 647 [hep-ph/9804253].

[5] CMS Collaboration, Measurement of the CP-violating weak phase $\phi_{s}$ and the decay width difference $\Delta \Gamma_{s}$ using the $B_{s}^{0} \rightarrow J / \psi \phi$ decay channel in pp collisions at $\sqrt{s}=8 \mathrm{TeV}$, Phys. Lett. $B 713$ (2012) 378, [hep-ex/1508 . 07527].

[6] A. S. Dighe, I. Dunietz, H. J. Lipkin, and J. L. Rosner, Angular distributions and lifetime differences in $B_{s}^{0} \rightarrow J / \psi \phi$ decays, Phys. Lett. B 369 (1996) 144, [hep-ph/9511363].

[7] Particle Data Group, M. Tanabashi et al., Review of particle physics, Phys, Rev. D 98 (2018) 030001.

[8] A. Lenz and G. Tetlalmatzi-Xolocotzi, Model-independent bounds on new physics effects in non-leptonic tree-level decays of B-mesons, [hep-ph/1912.07621]. 